The University of Maine

\title{
DigitalCommons@UMaine
}

Psychology Faculty Scholarship

Psychology

1988

\section{Application of Imagery Theory to Sport Psychology: Some Preliminary Findings}

Jeffrey E. Hecker

University of Maine - Main, jeff.hecker@umit.maine.edu

Linda M. Kaczor

Follow this and additional works at: https://digitalcommons.library.umaine.edu/psy_facpub

Part of the Psychology Commons

\section{Repository Citation}

Hecker, Jeffrey E. and Kaczor, Linda M., "Application of Imagery Theory to Sport Psychology: Some Preliminary Findings" (1988).

Psychology Faculty Scholarship. 11.

https://digitalcommons.library.umaine.edu/psy_facpub/11

This Article is brought to you for free and open access by DigitalCommons@UMaine. It has been accepted for inclusion in Psychology Faculty Scholarship by an authorized administrator of DigitalCommons@UMaine. For more information, please contact

um.library.technical.services@maine.edu. 


\title{
Application of Imagery Theory to Sport Psychology: Some Preliminary Findings
}

\author{
Jeffrey E. Hecker and Linda M. Kaczor \\ University of Maine
}

\begin{abstract}
Bioinformational theory has been proposed by Lang (1979a), who suggests that mental images can be understood as products of the brain's information processing capacity. Imagery involves activation of a network of propositionally coded information stored in long-term memory. Propositions concerning physiological and behavioral responses provide a prototype for overt behavior. Processing of response information is associated with somatovisceral arousal. The theory has implications for imagery rehearsal in sport psychology and can account for a variety of findings in the mental practice literature. Hypotheses drawn from bioinformational theory were tested. College athletes imagined four scenes during which their heart rates were recorded. Subjects tended to show increases in heart rate when imagining scenes with which they had personal experience and which would involve cardiovascular activation if experienced in real life. Nonsignificant heart rate changes were found when the scene involved activation but was one with which subjects did not have personal experience.
\end{abstract}

Mental practice has been defined as rehearsal of a physical task in the absence of observable movement (Corbin, 1972; Richardson, 1967). It typically involves the use of imagery, but the term has been used to include other procedures such as reading a description or watching a film of a task properly executed (Weinberg, 1982). The focus of this paper is on imagery rehearsal, a specific mental practice technique (Suinn, 1982). Several authors have reviewed the literature on mental practice and most have offered the cautious conclusion that mental practice usually helps improve performance on motor tasks (Corbin, 1972; Feltz \& Landers, 1983; Kaczor, 1987; Richardson, 1967; Suinn, 1985; Weinberg, 1982). In addition, some features of successful mental practice are beginning to emerge. Suinn (1985), for example, identified the following factors as being related to successful mental practice: (a) It appears to be associated with below threshold muscular activity during practice. (b) It appears to be most effective when used in conjunction with physical practice. (c) It tends to work bet-

Requests for reprints should be sent to Jeffrey E. Hecker, Department of Psychology, 310 Little Hall, University of Maine, Orono, ME 04469. 
ter with experienced performers. (d) Mental practice has its strongest effect on simple motor tasks. (e) Distributed practice appears to be more effective than massed practice.

Sport psychology has been criticized as being theory poor (Mackay, 1981; Wollman, 1986) and the mental practice literature is no exception (Kaczor, 1987). A sound theoretical basis for the relationship between imagery rehearsal and improved athletic performance that can accommodate the extant empirical findings has not been developed to date. It has recently been suggested that there are generally three theoretical formulations of mental practice effects in the sport science literature: symbolic learning, psychoneuromuscular, and attention-arousal set (Feltz \& Landers, 1983; Vealey, 1987). Each of these formulations has been found lacking (Kaczor, 1987).

The symbolic learning view was originally put forth by Sackett (1935), who speculated that symbolic rehearsal, the acquisition and subsequent rehearsal of symbols that represent patterns of overt movements, will facilitate the learning of skills in which cognitive factors are predominant. Tasks primarily characterized by muscle movement would not be strongly influenced by symbolic rehearsal, according to Sackett. As a theory of imagery rehearsal, this view has some serious problems. First, although it can account for the finding that mental practice facilitates early skill acquisition, the symbolic learning view has little to say about performance enhancement of well learned athletic skills. Second, the division of tasks into cognitive and motor components appears to be artificial (Richardson, 1967).

The psychoneuromuscular formulation holds that during imagery of overt motor acts there are minute innervations, identical in pattern but weaker in magnitude, of the involved muscles. During mental practice the learner obtains visual and kinesthetic feedback that is used to make adjustments in motor performance (Richardson, 1967). This view and variants of it have alternatively been referred to as the muscle memory theory (Vealey, 1987), the feedback theory (Corbin, 1972), the muscle potential hypothesis (Mackay, 1981), the neuromuscular feedback theory (Harris \& Robinson, 1986), and the mirror hypothesis (Feltz \& Landers, 1983). There is considerable support for the proposition that imagery rehearsal is accompanied by small but measurable activations of visceral and motor responses appropriate to the task imagined (Anderson, 1981). The psychoneuromuscular view is weak in explaining how feedback results in improved performance. There are several unanswered questions with regard to feedback. For example, are the adjustments in motor performance the result of conscious cognitive processes or do they occur without conscious awareness? If unnecessary or interfering muscles are activated, what mechanism signals that this was an inappropriate response? The psychoneuromuscular view is perhaps best considered a description of an important aspect of effective imagery rehearsal, namely visceral and muscular activation, rather than an explanation of the processes involved in improved performance.

The attention-arousal set formulation attempts to incorporate both cognitive and physiological aspects of imagery rehearsal (Vealey, 1987). In this view, it is suggested that during imagery rehearsal an athlete may learn to set his or her physiological arousal at an optimal level (Feltz \& Landers, 1983). Cognitively, imagery may help bring attention to task relevant thoughts and away from 
task-irrelevant ones which may disrupt performance (Feltz \& Landers, 1983). Because the attention-arousal set formulation has not been explicated in detail, it is difficult to make a priori predictions based upon it. While intuitively appealing (Feltz \& Landers, 1983), this view has not been empirically examined. Critical evaluation therefore is impossible.

The purpose of the present paper is to explicate a recently proposed theory of emotional imagery and to present some preliminary findings that demonstrate its application to sport psychology. Bioinformational theory has recently been proposed by Lang (1977, 1979a, 1985), who suggests that mental images can be understood as products of the brain's information processing capacity. He argues that an image is "a finite information structure which can be reduced to specific propositional units" (Lang, 1979b, p. 109). Imagery involves activation of a network of propositionally coded information stored in long-term memory. These propositions can be organized into at least two categories of information: information about the stimulus characteristics of the imagined situation, and information about the physiological and overt behavioral responses. This information network is considered to be a prototype for overt behavior. The prototype can be processed by internally generating prototype-matching information (e.g., through imagery). Processing occurs when a critical number of propositions are accessed. Imagining oneself throwing a baseball, for example, involves activating stimulus propositions that would include descriptors of the texture and feel of the ball in one's hand and associated visual stimuli (e.g., the catcher's glove). Response propositions would include muscular changes in the arm and shoulder as well as cardiovascular activation. Vivid imagery involves processing of stimulus and response propositions.

Processing of response propositions is associated with detectable psychophysiological changes. Changes in muscle tension, for example, can provide a real-time indicator of response information processing. Several factors influence whether or not subjects show psychophysiological changes during imagery. First, an image script (i.e., description of the scene to be imagined) needs to describe a situation which, if encountered in vivo, would involve physiological activity (Lang, Kozak, Miller, Levin, \& McLean, 1980). Second, activation of response elements is facilitated by instructions that explicitly direct subjects to experience the image physiologically. This effect is strongest when imagery scripts contain references to muscular and visceral responses (Lang, Levin, Miller, \& Kozak, 1983). Third, self-rated "'good” imagers are better able to generate physiological changes during imagery (Levin, Cook, \& Lang, 1982). Fourth, activation of response information is more likely when scenes are personally relevant to the subject (i.e., subject has experience with the imagined scene) (Miller, et al., 1986; Port \& Hecker, 1987).

According to bioinformational theory, for imagery rehearsal to affect athletic performance, response propositions, which provide a prototype for the overt motor act, must be activated so they can be altered in some way. For example, extraneous response information (e.g., motor movements, anxiety-related responses) may become detached from the network during processing and response characteristics important for proper execution may be strengthened. A bioinformational analysis of imagery rehearsal may help explain some of the trends in the mental practice literature. 
There is evidence in the extant mental practice literature to suggest that response processing during imagery facilitates improvement in task performance. First, it has been suggested based on correlational data that imagery rehearsal is more powerful when subjects take an internal as opposed to an external perspective when rehearsing a skill in imagination (Mahoney \& Avener, 1977; Weinberg, 1982). Internal imagery involves imagining oneself actually performing the skilled task (i.e., first person) whereas in external imagery one observes oneself in imagination performing the task (i.e., third person). From a bioinformational analysis, internal imagery would facilitate processing of response information since it requires the subject to actually perform the task in imagination. Because, as we have suggested, processing of response information is necessary in order to change the prototype for overt behavior stored in long-term memory, superiority of internal imagery would be consistent with the theory. Evidence consistent with the hypothesis that internal imagery facilitates response processing is provided by Hale (1982) and Harris and Robinson (1986). Subjects using internal imagery showed more EMG activity than did subjects using external imagery in both studies. Hale explicitly related EMG concomitants during internal imagery to Lang's response information processing.

In a similar vein, Suinn (1985) has noted that instructing subjects to experience an imagined scene as if they were actually carrying it out (i.e., feel muscular and emotional reactions) facilitates improvement with visuo-motor behavior rehearsal (VMBR), his method of imagery rehearsal. Once again, this is consistent with bioinformational theory because it suggests that encouraging subjects to process response information during imagery is associated with improved performance. Richardson (1967) and Corbin (1972) have noted in their reviews of the literature that successful imagery rehearsal is often associated with below threshold changes in muscular activity. This provides further evidence that mental practice involves processing of response information. These small changes in musculature are held to be the efferent output of response information processing (Lang, 1979a). Suinn and Dickinson (1978) found that skiers asked to "think about" skiing through gates showed no changes in EMG activity in the appropriate muscle groups. When asked to use imagery rehearsal, however, these same athletes showed increases in EMG activity. Hall and Erffmeyer (1983) found that the subjects who demonstrated the largest gains in free-throw accuracy following VMBR with modeling reported feeling muscle activity during imagery.

A third finding that can be accounted for using bioinformational theory is that experienced athletes tend to benefit more from rehearsal than do novices (Feltz \& Landers, 1983; Noel, 1980; Weinberg, 1982). Experienced athletes have already learned the motor sequence associated with successful performance. This information would, according to the theory, be stored as a network of propositions in long-term memory. Imagery rehearsal would involve processing of the memory network. The novice has no prototype for skillful performance in memory. Hence, imagery would involve activation of related information that would likely be connected with superfluous and perhaps interfering response propositions that would be rehearsed as well.

Bioinformational theory, of course, is not the only formulation that can account for these findings. Some of the data are consistent with the symbolic learning, psychoneuromuscular, and attention-arousal views. The strength of Lang's theory, however, lies in its heuristic value since it provides a conceptual model 
that can guide research into imagery rehearsal. In a 1982 article in this journal, Hale recognized the potential of bioinformational theory for sport psychology. Unfortunately, in the intervening years few empirical tests of the theory have emerged.

The present study represents our preliminary efforts at applying the theory to imagery rehearsal in sport psychology. Two hypotheses drawn from bioinformational theory were tested. These hypotheses dealt with the conditions under which subjects would activate response information during imagery. Activation of response information was operationally defined as a significant increase in heart rate above baseline level. First, it was predicted that varsity athletes would show increases in heart rate when imagining situations with which they had personal experience and which involved physiological responsivity. Conversely, nonsignificant changes in heart rate were expected when subjects imagined scenes with which they had no personal experience or which did not call for cardiovascular activation. Second, subjects were expected to vary in the heart rate changes they demonstrated during imagery, and these changes were expected to be related to self-rated imagery ability. The last hypothesis is based on the finding that there are individual differences in the tendency to process response information during imagery (Levin et al., 1982).

\section{Method}

\section{Subjects and Apparatus}

Subjects were 19 members of a varsity NCAA Division I-AA women's softball team. The data were collected in the context of ongoing consultation between the authors and the team. Participation was voluntary. Data from one subject were lost, due to experimental error.

Heart rate was recorded using the Biofeedback Microlab, HMR software, and an Apple IIe microcomputer. A photoelectric plethysmograph was attached to the subject's right earlobe so that variations of the amount of light transmitted through the tissues associated with each heart beat were detected. Heart rate, in beats per minute, was recorded by an experimenter in a separate room.

\section{Procedure}

The purpose of the study was explained to subjects in a group meeting. They were told their heart rate activity would be recorded as they imagined various scenes. Subjects completed the Questionnaire Upon Mental Imagery (QMI), a 35-item self-report measure of imagery ability (Sheehan, 1967) during this meeting. Imagery assessments were conducted individually. Subjects were seated in a comfortable chair and listened to prerecorded instructions through stereo headphones. After a brief overview of the procedures, subjects were presented with 6 minutes of relaxation instructions so that heart rate would achieve a stable baseline level. Four scenes were then presented. Prior to each scene there were 2 minutes of silence during which subjects were instructed to relax. The scene was then described. Scene presentation varied from 36 to 67 seconds. After each description, subjects were given 45 seconds to imagine the scene. They were then instructed to stop imagining and concentrate on relaxation for 45 seconds. Subjects were then asked to rate the vividness of their imagery on a 9-point scale 
$(0=$ no image; $8=$ lifelike image $)$. Imagery vividness ratings were made verbally and were recorded by the experimenter in an adjoining room.

The four scenes were as follows: neutral (sitting on a porch relaxing), action (doing bench presses in the weight room), athletic anxiety (batting at a critical point in a game), and fear (being in a plane that is tumbling toward the ground). Excerpts from each script are reproduced below:

\section{Neutral}

You are sitting in a lawn chair on your porch on a summer afternoon. Leaning back, relaxed, you feel a soft warm breeze blowing across the porch. .. Comfortable and content you are so relaxed you hardly move. . .

\section{Action}

You are in the weight room about halfway through your workout. . . You lift the bar out of the holder and slowly lower it to your chest. . . As you proceed, you feel your heart begin to pound. Your pectoral and tricep muscles begin to strain. . .

\section{Athletic Anxiety}

You are walking to the batter's box during the final inning of a home game... Your heart is pounding and your mouth is dry. You want so much to get a hit because you made the error that allowed the other team to tie the score... You feel butterflies in your stomach. . .

\section{Fear}

You are in a jet plane cruising at a very high altitude. Suddenly, there is a loud booming sound like an explosion. . . Your heart pounds wildly in your chest and your muscles go stiff. The sound of the screaming engines and the cries of the other passengers is deafening. . .

Scenes were always presented in the same order: neutral, action, athletic anxiety, fear. All scenes contained response propositions with the exception of the neutral scene. The action, athletic anxiety, and fear scenes each contained two propositions referring directly to cardiovascular responses. The content of the four scenes was chosen to represent combinations of experience with the situation and degree of cardiovascular activation. The neutral scene was one most subjects were expected to be somewhat familiar with and one that involved minimal activity. The action and athletic scenes involved cardiovascular activation. These scenes were also similar to situations experienced by the athletes. The fear scene was one that would involve cardiovascular activation but with which the subjects had not had experience.

A 30-sec sample of subjects' heart rate was recorded during the 2nd minute of the 2-min baseline period prior to each scene (baseline), as the scene was read (read), during the 45-sec imagery period (image), and during the 45-sec recovery period (recovery) when subjects were asked to stop imagining the scene. 


\section{Results}

\section{Heart Rate}

Heart rate data were examined for each scene individually by means of repeated measures analysis of variance across baseline, read, image, and recovery periods. Heart rate for each 30 -sec sample, converted to mean beats per minute, were used in the analyses. Significant period effects were found for the neutral, $F(3,51)=3.11, p<.05$, action, $F(3,51)=9.62, p<.001$, and athletic anxiety, $F(3$, $51)=4.65, p<.01$ scenes. A nonsignificant period effect was found for the fear scene. Multiple $t$ tests comparing heart rate during read, image, and recovery periods with baseline heart rate indicated significantly higher heart rate during read and image periods for the neutral, $t(17)=2.82, p<.05 ; t(17)=3.15, p<.05$; action, $t(17)=3.75, p<.01 ; t(17)=3.56, p<.01$; and athletic anxiety, $t(17)=2.72$, $p<.05 ; t(17)=3.00, p<.05$ scenes. For each of these scenes, heart rate returned to levels not significantly different from baseline by the recovery period. Mean heart rates for the image periods for each scene were compared using a repeated measures analysis. Heart rate during imagery differed significantly across the four scenes, $F(3,48)=6.95, p<.005$.

Individual comparisons using $t$ tests indicated that mean heart rates for the image periods were significantly higher during the action and athletic anxiety scenes compared with the fear scene, $t(17)=4.52, p<.005 ; t(17)=2.57, p<.05$. Similarly, mean heart rate during the imagery period for the action scene was significantly higher than during the neutral scene, $t(17)=2.03, p<.05$. Heart rate during imagery of the athletic anxiety scene was higher than during imagery of the neutral scene but this difference failed to reach conventional significance levels, $t(17)=1.58, p<.07$. Heart rate data for each scene are graphed in Figure 1, which clearly illustrates that subjects' heart rate increased during read and image periods and returned to near baseline levels during the recovery period for the action and athletic anxiety scenes. This trend is not evident for the fear scene and is less clear for the neutral scene. Mean heart rate in beats per minute and standard deviations are presented in Table 1.

\section{Imagery Vividness}

Imagery vividness ratings were examined by repeated measures analysis of variance across the four scenes. A significant scene effect was discovered. Subjects rated images of the action and athletic anxiety scenes as significantly more vivid than the neutral, $t(17)=2.61, p<.05 ; t(17)=3.04, p<.05$; and fear, $t(17)=3.43, p<.01 ; t(16)=3.77, p<.01$, scenes. Vividness ratings for the neutral and fear scenes did not differ significantly. Mean vividness ratings and standard deviations are included in Table 1.

\section{Self-Report Imagery Ability}

Subjects' total scores on the QMI were used as the measure of self-reported imagery ability. Although the QMI has seven subscales, factor analytic studies 

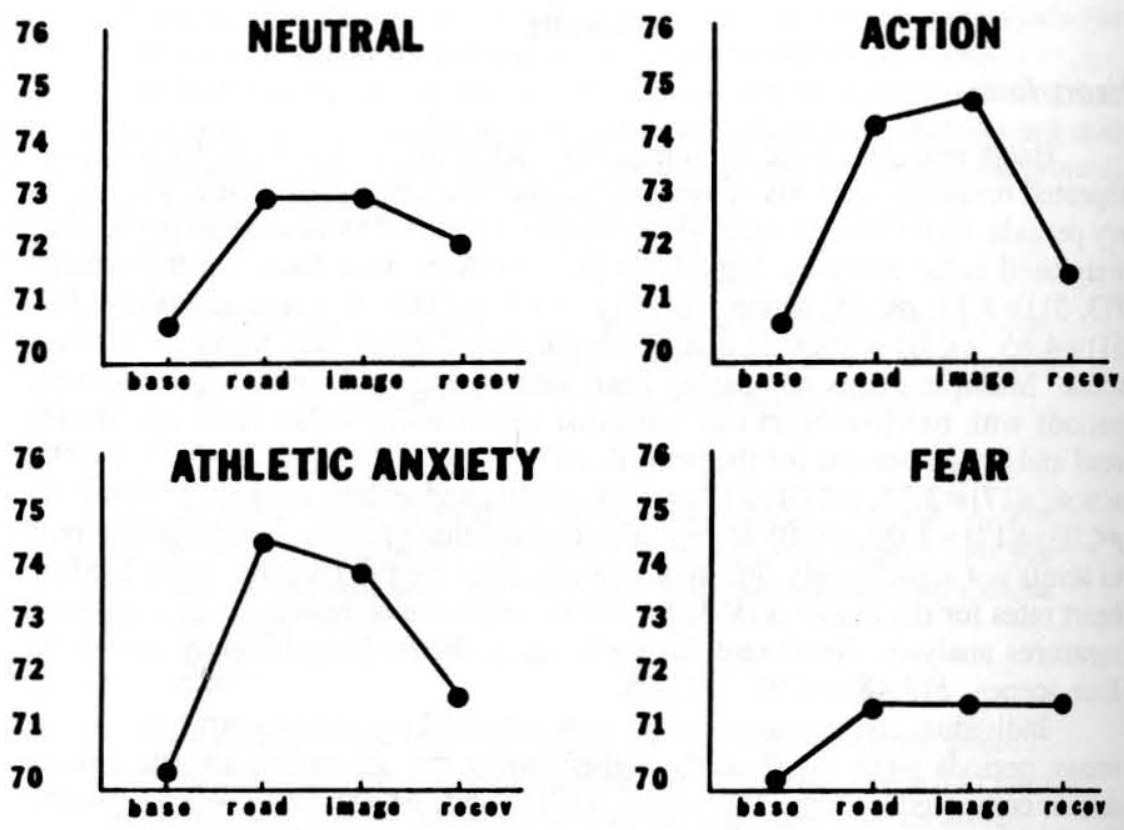

Figure 1 - Mean heart rate during baseline, read, image, and recovery periods for each scene.

Table 1

Heart Rate for Each Period of Each Scene and Imagery Vividness Ratings: Means and Standard Deviations

\begin{tabular}{lccccc}
\hline & \multicolumn{5}{c}{ Period } \\
\cline { 2 - 6 } Scene & Baseline & Read & Image & Recovery & Vividness \\
& & & & & \\
\hline \multirow{2}{*}{ Neutral } & 70.54 & 72.82 & 73.21 & 71.77 & 5.72 \\
& $(9.68)$ & $(9.80)$ & $(9.10)$ & $(8.21)$ & $(1.02)$ \\
Action & 70.63 & 74.64 & 75.09 & 71.29 & 6.39 \\
& $(8.99)$ & $(10.25)$ & $(9.30)$ & $(8.57)$ & $(1.04)$ \\
Athletic anxiety & 70.13 & 74.27 & 74.15 & 71.39 & 6.78 \\
& $(9.26)$ & $(9.47)$ & $(8.75)$ & $(9.47)$ & $(1.40)$ \\
Fear & 70.08 & 71.34 & 71.68 & 71.47 & 4.94 \\
& $(9.77)$ & $(8.81)$ & $(7.82)$ & $(9.10)$ & $(1.71)$
\end{tabular}

Note. Standard deviations appear in parentheses. 
have indicated that most of the variance can be accounted for by a single vividness factor (Richardson, 1967). Subjects' mean QMI score was 70.16 $(S D=18.24)$, with a range from 44 to 99 . As a group, the athletes indicated above average imagery ability when compared with relevant normative standards. Miller et al. (1986), for example, found an average score of $87.33(S D=24.57)$ in a sample of 1,520 college students (lower scores indicate more vivid imagery). In fact, only three of the athletes in this study scored above the mean of the Miller et al. sample.

The relationship between subjects' scores on the QMI and heart rate activity during imagery was examined by correlating heart rate responses during imagery with QMI scores. The difference between heart rate during imagery and heart rate during baseline was used as the measure of heart rate response. Nonsignificant correlations between QMI scores and heart rate change scores for each scene were found. Self-rated imagery ability therefore was not an accurate predictor of activation of response information. Similarly, QMI scores were not correlated with imagery vividness ratings for any scene. The fact that the QMI was not related to other measures of imagery in this study may have been due to the relatively narrow range of self-reported imagery ability for these athletes.

\section{Discussion}

Athletes' cardiac activity during imagery of the various scenes provides some support for the application of bioinformational theory to sport psychology. Activation of response information (i.e., heart rate increases) occurred during imagery of scenes with which subjects had some familiarity and which involved physiological activation (i.e., action and athletic anxiety scenes), and imagery for these scenes was perceived to be more vivid by subjects. During imagery of the fear scene, response information processing did not occur (i.e., nonsignificant increase in heart rate). This is consistent with the theory since subjects did not have experience with this situation and therefore presumably did not have a prototype for overt responding stored in long-term memory.

The heart rate data for the neutral scene did not correspond as clearly with theoretical predictions. Despite the fact that the script for this scene did not include cardiovascular response propositions, subjects showed a significant increase in mean heart rate during imagery. Although this effect was not as strong as it was for the action and athletic anxiety scenes, it still needs to be accommodated. That the neutral scene script did not contain response propositions does not necessarily mean subjects did not include response details in their imagery. Anderson and Borkovec (1980) employed a content analysis procedure to examine subjects' narrative descriptions of their imagery. They found that subjects who read scripts containing stimulus-plus-response propositions included more response references in their narratives than did subjects who read stimulus-only scripts. Interestingly, however, they also found that a large percentage of stimulus-only subjects included response details in their imagery. It is possible, therefore, that in the present study subjects included response information in their imagery of the neutral scene. 
The fact that subjects showed a significant increase in heart rate during imagery of this scene, and that the increase was less than for imagery of the action and athletic anxiety scenes, is consistent with Anderson and Borkovec's results. These investigators found a positive correlation between heart rate responsiveness and number of response propositions in subjects' narratives. Presumably, in our study, more response propositions were included in the action and athletic anxiety as compared to the neutral scene. Without content analyses of subjects' imagery descriptions, this presumption cannot be tested. It would seem beneficial that in future investigations a content analysis system for assessing imaginal detail, such as the one described by Anderson (1981), be employed.

Bioinformational theory can be used to integrate previous findings in the sport psychology literature and provides a useful paradigm to guide future efforts to study the relationship between imagery rehearsal and improved athletic performance. The results of the present study indicate that experience level and the inclusion of response information in imagery scripts are associated with changes in measurable psychophysiological variables. The next step, of course, is to demonstrate in an a priori fashion that subthreshold changes in muscular and cardiovascular systems during imagery, which are held to be indicators of response information processing in the brain, are necessary for improved performance as predicted from the theory. An experiment designed to test this hypothesis is under way in our laboratory.

\section{References}

Anderson, M.P. (1981). Assessment of imaginal processes: Approaches and issues. In T.V. Merluzzi, C.R. Glass, \& M. Genest (Eds.), Cognitive assessment (pp. 149-187). New York: Guilford Press.

Anderson, M.P., \& Borkovec, T.D. (1980). Imaginal processing and fear reduction during repeated exposure to two types of phobic imagery. Behavior Research and Therapy, 18, 537-540.

Corbin, C. (1972). Mental practice. In W. Morgan (Ed.), Ergogenic aids and muscular performance (pp. 93-118). New York: Academic Press.

Feltz, D.L., \& Landers, D.M. (1983). The effects of mental practice on motor skill learning and performance: A meta-analysis. Journal of Sport Psychology, 5, 25-27.

Hale, B.D. (1982). The effects of internal and external imagery on muscular and ocular concomitants. Journal of Sport Psychology, 4, 379-387.

Hall, E.G., \& Erffmeyer, E.S. (1983). The effect of visuo-motor behavior rehearsal with videotaped modeling on free throw accuracy of intercollegiate female basketball players. Journal of Sport Psychology, 5, 343-346.

Harris, D.V., \& Robinson, W.J. (1986). The effects of skill level on EMG activity during internal and external imagery. Journal of Sport Psychology, 8, 105-111.

Kaczor, L. (1987). Mental practice: A review of theory and research. Unpublished manuscript.

Lang, P.J. (1977). Imagery in therapy: An information processing analysis of fear. Behavior Therapy, 8, 862-886.

Lang, P.J. (1979a). A bio-informational theory of emotional imagery. Psychophysiology, 16, 495-512. 
Lang, P.J. (1979b). Language, image, and emotion. In K. Pliner, K.R. Blankenstein, \& I.M. Speigal (Eds.), Advances in the study of communication and affect: Perception of emotion in self and others (pp. 107-117). New York: Plenum Press.

Lang, P.J. (1985). The cognitive psychophysiology of emotion: Fear and anxiety. In A.H. Tuma \& J.D. Maser (Eds.), Anxiety and the anxiety disorders (pp. 131-170). Hillsdale, NJ: Erlbaum.

Lang, P.J., Kozak, M.J., Miller, G.A., Levin, D.N., \& McLean, A. (1980). Emotional imagery: Conceptual structure and pattern of somato-visceral response. Psychophysiology, 17, 179-192.

Lang, P.J., Levin, D.N., Miller, G.A., \& Kozak, M.J. (1983). Fear behavior, fear imagery, and the psychophysiology of emotion: The problem of affective response integration. Journal of Abnormal Psychology, 92, 276-306.

Levin, D.N., Cook, E.W., \& Lang, P.J. (1982). Fear imagery and fear behavior: Psychophysiological analysis of clients receiving treatment for anxiety disorders. Psychophysiology, 19, 571-572.

Mackay, D.G. (1981). The problem of rehearsal or mental practice. Journal of Motor Behavior, 13, 274-285.

Mahoney, M.J., \& Avener, M. (1977). Psychology of the elite athlete: An exploratory study. Cognitive Therapy \& Research, 2, 135-141.

Miller, G.A., Levin, D.N., Kozak, M.J., Cook, E.W., McLean, A., \& Lang, P.J. (1986). Emotional imagery: Effects of individual differences in perceptual imagery on physiological response. Unpublished manuscript.

Noel, R.C. (1980). The effect of visuo-motor behavior rehearsal on tennis performance. Journal of Sport Psychology, 2, 221-226.

Port, E., \& Hecker, J.E. (1987). [Fear classification, imagery training, and personal meaningfulness of imagery: A test of bioinformational theory.] Unpublished raw data.

Richardson, A. (1967). Mental practice: A review and discussion. Part II. Research Quarterly, 38, 263-273.

Sackett, R.S. (1935). The relationship between the amount of symbolic rehearsal and retention of a maze habit. Journal of General Psychology, 13, 113-128.

Sheehan, P.W. (1967). A shortened form of Betts' questionnaire upon mental imagery. Journal of Consulting Psychology, 23, 386-389.

Suinn, R.M. (1982). Imagery in sports. In A. Sheikh (Ed.), Imagery, current theory, research, and application (pp. 507-534). New York: Wiley.

Suinn, R.M. (1985). Imagery rehearsal applications to performance enhancement. The Behavior Therapist, 8, 155-159.

Suinn, R.M., \& Dickinson, A. (1978). Imagery rehearsal as a form of motor skill. Unpublished manuscript.

Vealey, R.S. (1987, June). Imagery training for performance enhancement. Paper presented at the Sports Psychology Institute, Portland, ME.

Weinberg, R.S. (1982). The relationship between mental preparation strategies and motor performance: A review and critique. Quest, 33, 728-734.

Wollman, N. (1986). Research on imagery and motor performance: Three methodological suggestions. Journal of Sport Psychology, 8, 124-128.

Manuscript submitted: September 1, 1987

Revision received: January 11, 1988 